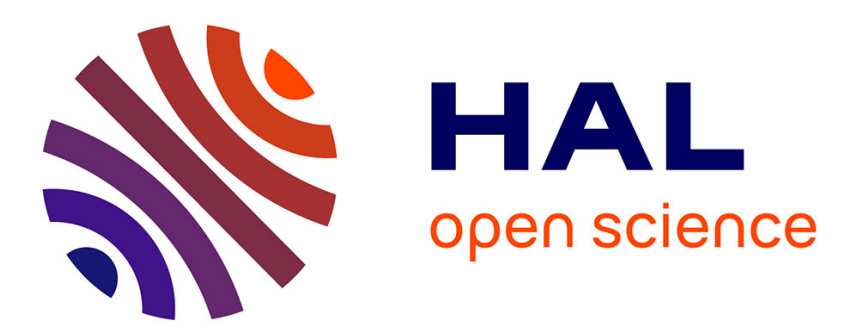

\title{
Explanation of the huge difference between vacuum energy and dark energy in the theory of the dynamic medium of reference \\ Olivier Pignard
}

\section{- To cite this version:}

Olivier Pignard. Explanation of the huge difference between vacuum energy and dark energy in the theory of the dynamic medium of reference. Physics Essays, 2021, 34 (1), pp.61-67. 10.4006/08361398-34.1.61 . hal-03147654

\author{
HAL Id: hal-03147654 \\ https://hal.science/hal-03147654
}

Submitted on 20 Feb 2021

HAL is a multi-disciplinary open access archive for the deposit and dissemination of scientific research documents, whether they are published or not. The documents may come from teaching and research institutions in France or abroad, or from public or private research centers.
L'archive ouverte pluridisciplinaire HAL, est destinée au dépôt et à la diffusion de documents scientifiques de niveau recherche, publiés ou non, émanant des établissements d'enseignement et de recherche français ou étrangers, des laboratoires publics ou privés. 


\title{
Explanation of the huge difference between vacuum energy and dark energy in the theory of the dynamic medium of reference
}

Olivier Pignard $^{\text {a) }}$

16 Boulevard du Docteur Cathelin, 91160 Longjumeau, France

(Received 25 October 2020; accepted 9 January 2021; published online 3 February 2021)

\begin{abstract}
The object of this article is to present the vacuum energy and the dark energy within the framework of the theory of the dynamic medium of reference and to explain the phenomenal difference between the two energies. The dynamic medium is made up of entities (called gravitons) whose vectorial average of speed determines the speed of the flux of the medium at each point in space. It is shown that inside the horizon of black holes (defined by the Schwarzschild radius), the speed of flux is greater than the speed of light, which means that the gravitons themselves have a higher speed to that of light. The quantum entanglement of two photons which propagate in two opposite directions is due to a connection made by gravitons. It is therefore proposed that the gravitons move at the speed $V_{G} \approx \frac{R_{\text {univers }}}{t_{\text {Planck }}} \approx 2.4 \times 10^{69} \mathrm{~m} / \mathrm{s}$, which makes it possible to guarantee the entanglement of the two photons whatever their position in the Universe and the impossibility to measure the time taken by the influence of a photon to arrive at its twin photon because it is

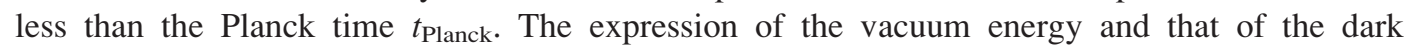
energy within the framework of the theory of the dynamic medium of reference is established. The two expressions $E_{\text {vacuum }}$ and $E_{\text {dark }}$ and the value of the speed of the most distant galaxies $V_{\text {galaxy }}$ make it possible to calculate an approximate value of the speed of the gravitons $V_{G} \approx V_{\text {galaxy }} \sqrt{\frac{E_{\text {vacum }}}{E_{\text {dark }}}} \approx 2.8 \times 10^{69} \mathrm{~m} / \mathrm{s}$. This value of the speed of the graviton is very close to that obtained by the quantum entanglement of two photons, which reinforces the existence of nonmaterial entities going at these phenomenal speeds. @ 2021 Physics Essays Publication.
\end{abstract}

[http://dx.doi.org/10.4006/0836-1398-34.1.61]

Résumé: L'objet de cet article est de présenter l'énergie du vide et l'énergie noire dans le cadre de la théorie du milieu dynamique de référence et d'expliquer l'énorme différence entre les deux énergies. Le milieu dynamique est constitué d'entités (appelés gravitons) dont la moyenne vectorielle des vitesses détermine en chaque point de l'espace la vitesse des flux du milieu. Il est montré qu'à l'intérieur de l'horizon des trous noirs (défini par le rayon de Schwarzschild) la vitesse des flux est supérieure à la vitesse de la lumière, ce qui entraine que les gravitons eux-mêmes possèdent une vitesse supérieure à celle de la lumière. L'intrication quantique de deux photons qui se propagent dans deux directions opposées est due à une connexion réalisée par les gravitons. Il est donc proposé que les gravitons se déplacent à la vitesse $V_{G} \approx \frac{R_{\text {univers }}}{t_{\text {Planck }}} \approx 2.4 .10^{69} \mathrm{~m} / \mathrm{s}$, ce qui permet de garantir l'intrication des deux photons quelle que soit leur position dans l'Univers et l'impossibilité de mesurer le temps mis par l'influence d'un photon pour arriver à son photon jumeau car il est inférieur au temps de Planck $t_{\text {Planck. }}$ L'expression de l'énergie du vide et celle de l'énergie noire dans le cadre de la théorie du milieu dynamique de référence sont établies. Les deux expressions $\mathrm{E}_{\mathrm{vide}}$ et $\mathrm{E}_{\text {noire }}$ et la valeur de la vitesse d'éloignement des galaxies les plus lointaines $\mathrm{V}_{\text {galaxie }}$ permettent de calculer une valeur approximative de la vitesse des gravitons $V_{G} \approx V_{\text {galaxie }} \sqrt{\frac{E_{\text {vide }}}{E_{\text {noire }}}} \approx 2.8 .10^{69} \mathrm{~m} / \mathrm{s}$. Cette valeur de la vitesse des gravitons est très proche de celle obtenue grâce à l'intrication quantique de deux photons, ce qui renforce l'existence d'entités nonmatérielles allant à ces vitesses phénoménales.

Key words: Vacuum Energy; Dark Energy; Dynamic Medium of Reference; Gravitons; Galaxy; Black Hole; Entanglement between Two Photons.

a) olivier_pacific@hotmail.fr 


\section{PHENOMENAL DIFFERENCE BETWEEN VACUUM ENERGY AND DARK ENERGY}

Vacuum energy is an underlying energy which exists everywhere in space, through the Universe.

A possible contribution to the vacuum energy is realized by the virtual particles that are seen as couples of particles which appear and annihilate in such a short time length that they cannot be observed. They would act like that in the whole Universe. Their behavior is codified in the time-energy relationship of the Heisenberg's uncertainty principle.

The effects of the vacuum energy can be experimentally observed in several phenomena and are supposed to influence the behavior of the Universe at the cosmological scale.

By using the superior limit of the cosmological constant, the vacuum energy has been estimated to $\mathbf{1 0}^{-\mathbf{9}}$ joules by cube meter ${ }^{1-3}$ and it corresponds to the dark energy.

Although according to quantum electrodynamics and stochastic electrodynamics, to be coherent with Lorentz's invariant principle and the order of magnitude of Planck's constant, it should have a value on the order of $\mathbf{1 0}^{\mathbf{1 1 3}}$ joules by cube meter. ${ }^{4,5}$

This huge divergence is called the "vacuum catastrophe."

\section{PRESENTATION OF THE DYNAMIC MEDIUM OF REFERENCE (DMR)}

The theory of the dynamic medium of reference ${ }^{6}$ (DMR) introduces a dynamic nonmaterial medium which is present in the whole Universe.

The characteristics of this medium are as follows:

- This medium enables one to deduce a Preferred Frame of Reference or rather a REFERENCE in the whole Universe and at all scales.

- This REFERENCE enables one to obtain a privileged time. The present moment is universal, that is to say the same in the whole Universe.

- This medium is also the medium of propagation of light.

- This medium verifies the principle of reciprocal action:

- The medium is distorted by matter and energy like the space-time of general relativity.

- The warping of this medium determines the trajectories of the particles (material particles and light particles).

The presence of a massive body creates a flux in the medium (centripetal that is to say directed toward the center of gravity of the massive body) of speed,

$$
V_{\text {flux }}=\sqrt{\frac{2 G M}{r}},
$$

and acceleration,

$$
\gamma_{\text {flux }}=\frac{G M}{r^{2}}
$$

where $r$ refers to the distance to the center of gravity of the massive body.

\section{A POSSIBLE DESCRIPTION OF THE DYNAMIC MEDIUM OF REFERENCE: THE GRAVITONS FIELD}

This part gives a possible description of the dynamic medium of reference: the gravitons field.

The theory of the dynamic medium of reference is based on Le Sage theory, but it adds many deep changes and evolutions.

Numerous scientists have studied Le Sage theory. Just to mention a few of them are Newton, Huygens, Leibniz, Euler, Laplace, Lord Kelvin, Maxwell, Lorentz, Hilbert, Darwin, Poincaré, and Feynman.

Henri Poincaré has studied this theory and written a synthesis in Science et Méthode. ${ }^{7}$

Poincaré sums up the principle of Le Sage theory as follows:

"It is proper to establish a parallel between these considerations and a theory proposed a long time ago in order to explain the universal gravitation. Let's suppose that, in the interplanetary spaces, very tiny particles move in all directions, with very high speeds. A single body in the space will not be affected, apparently, by the impact of these corpuscles, since these impacts are equally divided in all directions. But, if two bodies A and B are in the space, the body B will play the role of a screen and will intercept a part of these corpuscles which would have hit $\mathrm{A}$. Then, the impacts received by $\mathrm{A}$ in the opposite direction of the one of B, will not have compensation any longer, or will be imperfectly compensated, and they will push A toward B. Such is Le Sage theory."

It is possible to demonstrate rather easily that the "push" is inversely proportional to the square distance between the two bodies (like the Newton law).

One can also demonstrate that if the corpuscles are very tiny, the "push" is approximately proportional to the number of nucleons and so the mass of the body and not the apparent surface of the body.

Moreover, only a tiny fraction of corpuscles hits the atoms of the body, which explains that the "push" (the gravitational force) is so weak.

The main evolutions of the theory of the dynamic medium of reference versus Le Sage theory are the following:

- One must not use the notion of impact with the corpuscles.

- One must consider that the corpuscles are nonmaterial and constitute a medium.

- The total energy of an entity is the sum of its kinetic energy of translation and of its kinetic energy of rotation about itself.

- Fundamental law: conservation of the total energy of an entity: the total energy of one entity remains constant,

$$
E_{\text {total }}=E_{\text {translation }}+E_{\text {rotation }}=\text { constant. }
$$

Afterward I will call these entities as gravitons (but these gravitons have nothing to do with the graviton of spin 2 of quantum mechanics). 
If the total energy of the gravitons remains constant, then the gravitons do not give energy to the atoms of the Earth, and so they do not raise the temperature of the Earth.

It is postulated that the gravitons which interact with the atoms of the Earth lose some of their kinetic energy of translation which turns into kinetic energy of rotation. The gravitons which interact with the atoms of the Earth lose a part of their translation speed and win some rotation speed.

So the Earth would be a huge "transformer" of "standard gravitons" in "gravitons-spin."

This physical phenomenon has no effect on the Earth (at least not the elevation of temperature), but it has an effect on the medium.

The medium undergoes a centripetal flux due to the presence of the Earth.

Indeed, let us consider a reference frame at the surface of the Earth and an elementary volume linked to it.

If one measures the speed vectors of all the gravitons in this elementary volume, the average of the speed vectors gives a resulting speed vector which is centripetal (because the gravitons-spin coming from the ground have a smallest translation speed than the standard gravitons coming from the sky).

One can demonstrate that the acceleration of the flux has the following expression: $\overrightarrow{\gamma_{\text {flux }}}=-\frac{G M}{r^{2}} \overrightarrow{u_{r}}$ from which one can deduce that the centripetal speed of the flux at a distance $r$ from the center of gravity of the Earth of mass $M$ is equal to (measured in a reference frame $R$ linked to the Earth)

$$
\overrightarrow{C_{G / R}}=\frac{\sum_{i=1}^{N_{G}} \overrightarrow{V_{G / R}}}{N_{G}}=\overrightarrow{V_{\text {flux }}}=-\sqrt{\frac{2 G M}{r}} \vec{u}_{r} .
$$

The demonstration of the link between the acceleration and the speed of the flux is the following:

If we derivate the acceleration, we obtain

$$
\gamma_{\text {flux }}=\frac{d V}{d t}=\frac{d V}{d r} \frac{d r}{d t}=\frac{d V}{d r} \cdot V=\frac{1}{2} \frac{d}{d r}\left(V^{2}\right),
$$

so we have $V_{\text {flux }}^{2}=\int_{+\infty}^{r} 2 \gamma_{\text {flux }} d r$.

Using the expression $\gamma_{\text {flux }}=-\left(G M / r^{2}\right)$, we obtain $V_{\text {flux }}^{2}=-\int_{+\infty}^{r}\left(2 G M / r^{2}\right) d r=[2 G M / r]_{+\infty}^{r}=2 G M / r$.

Finally, we obtain the expression of the speed of the flux as $\overrightarrow{V_{\text {flux }}}=-\sqrt{(2 G M / r)} \overrightarrow{u_{r}}$.

Remark: the speed of the flux is zero at an infinite distance of the massive body creating the gravitational field $\left(V_{\text {flux }}=0\right.$ for $\left.r \rightarrow+\infty\right)$.

Definition of the Preferred Frame of Reference based on the entities constituting the medium.

Let us consider a Galilean referential $R$ (a laboratory) and an elementary volume linked to this referential.

In this very small volume, imagine that we can count the entities in it (gravitons) and we can also know the speed vector of each graviton, $\overrightarrow{V_{G / R}}$.

Knowing this, it is possible to compute the vectorial average of the speed vectors of the gravitons, $\overrightarrow{C_{G / R}}=$ $\sum_{i=1}^{N_{G}}\left(\overrightarrow{V_{G / R}}\right) / N_{G}$.
This resultant vector means that, at the center of this given elementary volume, the Preferred Frame of Reference moves at the speed $\overrightarrow{C_{G / R}}$ versus the referential $R$ and that the referential $R$ (the laboratory) moves at the speed $-\overrightarrow{C_{G / R}}$ versus the Preferred Frame of Reference (defined by the medium), i.e., versus the medium.

Figure 1 shows the distortion of the gravitons field created by the Earth.

The distortion of the gravitons field (the flux of the medium):

- is generated by the presence of the matter of the Earth;

- is always centripetal, i.e., radial, oriented toward the center of gravity of the Earth;

- is maximum at the surface of the Earth and decreases when going away from the Earth;

- has a constant modulus on every sphere whose center is the one of the Earth; and

- gives the impression to follow the Earth in its movement (whatever its speed) because it remains identical to itself.

\section{SPEED OF THE GRAVITONS}

\section{A. Speed of the flux of the gravitons inside the horizon of a black hole}

In general relativity, a stationary black hole, without rotation and without electric charge, is described by the metric of Schwarzschild, ${ }^{1}$

$$
\begin{aligned}
d s^{2}= & \left(1-\frac{2 G M}{c_{0}^{2} r}\right) c_{0}^{2} d t^{2}-\left(1-\frac{2 G M}{c_{0}^{2} r}\right)^{-1} d r^{2} \\
& -r^{2} d \vartheta^{2}-r^{2} \sin ^{2} \vartheta \cdot d \phi^{2}
\end{aligned}
$$

A black hole is bounded by the Schwarzschild radius $r_{S}=2 G M / c_{0}^{2}$ called the horizon of the black hole. Inside the horizon, nothing can escape from the black hole, not even light.

In the theory of the Dynamic Medium of Reference (DMR), a black hole generates a flux of centripetal gravitons (radial and directed toward the center of the black hole) whose speed has the following expression (Fig. 2): ${ }^{8}$

$$
V_{\text {flux }}=\sqrt{\frac{2 G M}{r}} .
$$

Beyond the horizon of a black hole $\left(r>r_{s}\right)$ given by the Schwarzschild radius $r_{S}=2 G M / c^{2}$, the speed of the flux of gravitons is lower than the speed of light.

However, inside the horizon of a black hole $\left(r<r_{s}\right)$, the speed of the flux of gravitons is greater than the speed of light.

Moreover, for a distance to the center of the black hole much less than the Schwarzschild radius $\left(r \ll r_{s}\right)$, the speed of the flux of gravitons is much greater than the speed of light $\left(V_{\text {flux }} \gg c\right)$.

For a distance $r$ corresponding to the Planck length $L_{P}=\sqrt{\left(G h /\left(2 \pi \cdot c^{3}\right)\right)}=1.616 \times 10^{-35} \mathrm{~m}$, the speed of the flux of gravitons is $V_{\text {flux }}=\sqrt{\left(2 G M / L_{P}\right)}=2.87 \times 10^{12} \sqrt{M}$. 


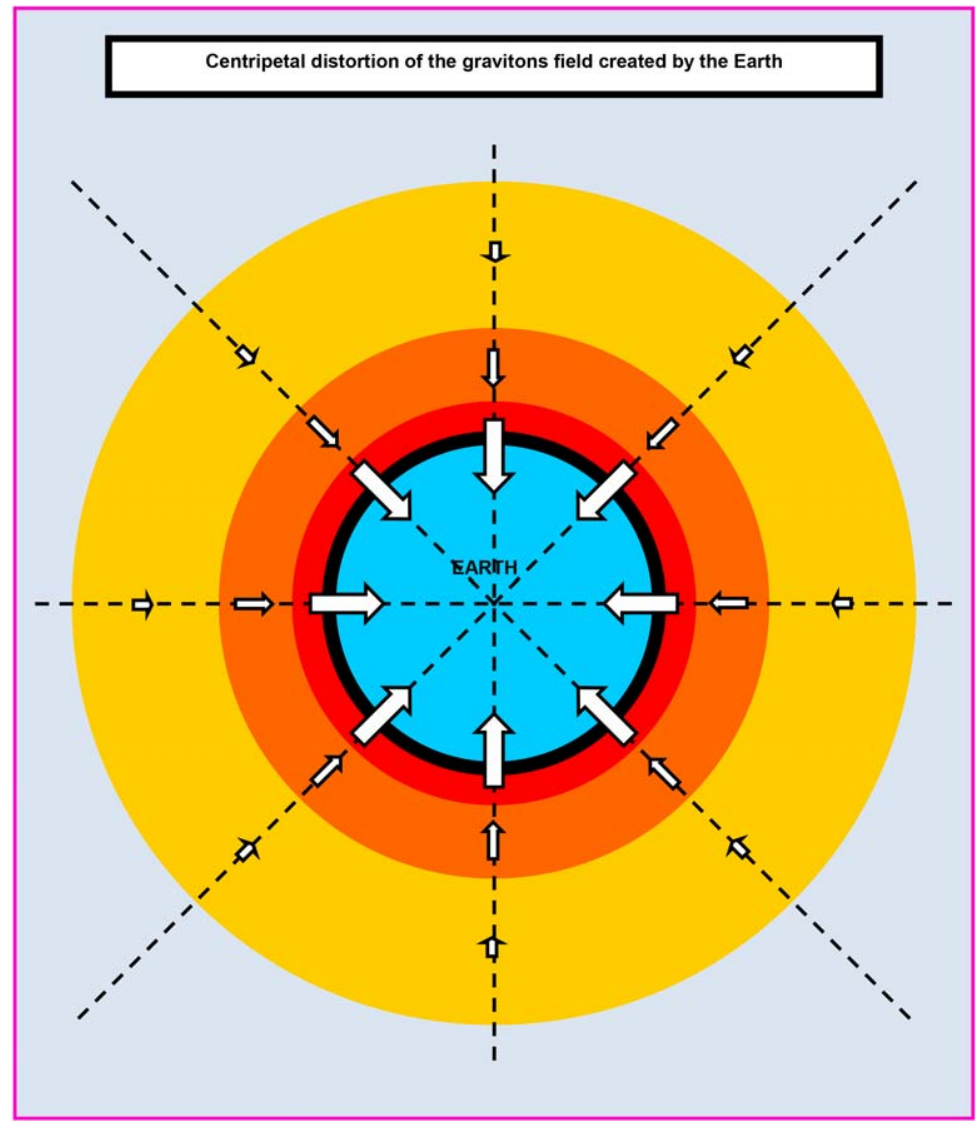

FIG. 1. (Color online) Distortion of the gravitons field due to the Earth.

For a black hole of a solar mass $\left(M s=2 \times 10^{30} \mathrm{~kg}\right)$, the speed of the flux of gravitons is $V_{\text {flux }}=4 \times 10^{27} \mathrm{~m} / \mathrm{s}$.

Inside the horizon of a black hole is the only place in the current Universe where the speed of the flux of gravitons is greater than the speed of light.

This result is of primary importance.

The speed vector of the flux of gravitons at a point $\mathrm{M}$ in space is given by the average of the speed vectors of all the gravitons located in the elementary volume centered in $M$,

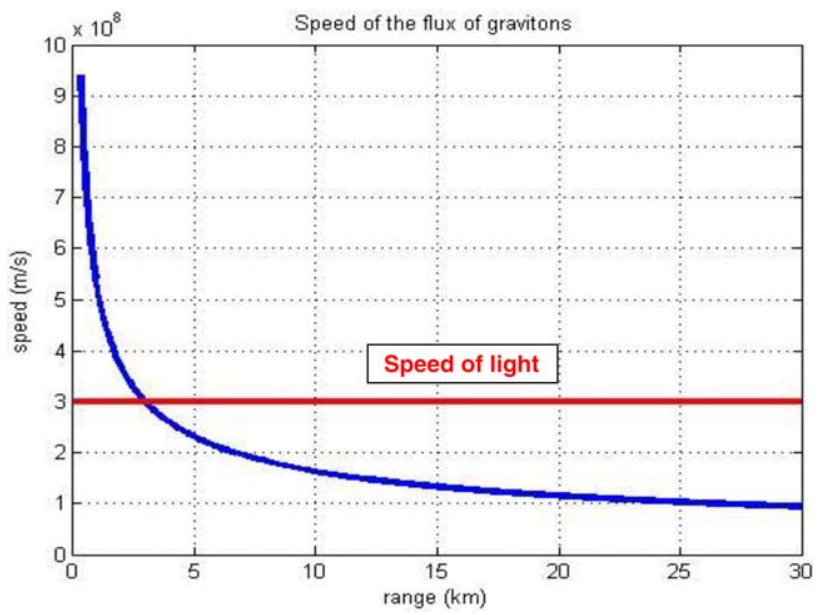

FIG. 2. (Color online) Speed of the flux of gravitons.

$$
\underset{V_{\text {flux } / R}}{\longrightarrow}(M)=\frac{\sum_{i=1}^{N_{G}} \overrightarrow{\mathrm{V}_{G / R}}}{N_{G}},
$$

where $R$ denotes any inertial reference frame and $N_{G}$ is the number of gravitons contained in the elementary volume centered in $M$.

If all the gravitons moved at the speed of light, the speed of the flux would be less than or equal to the speed of light (equality would be reached in the case where all the gravitons in a given elementary volume would move in the same direction).

A speed of the flux of gravitons much higher than the speed of light, which is the case at a distance very close to the center of a black hole $\left(r \ll r_{s}\right)$, implies that the speed of the gravitons themselves is much higher than the speed of light.

\section{B. Entanglement of two particles}

In The Ghost in the Atom, ${ }^{9}$ there is the following discussion about the EPR experiment and locality (p. 39):

"If locality is abandoned, it is possible to re-create a description of the microworld closely similar to that of the everyday world, with objects having a concrete independent existence in well-defined states and possessing complete sets of physical attributes. No need for fuzziness now. 
The trade-off is, of course, that nonlocal effects bring their own crop of difficulties; specifically, the ability for signals to travel backwards into the past. This would open the way to all sorts of causal paradoxes."

It is essential to be aware that all these difficulties are only in Einstein's vision, i.e., the special relativity (and general relativity).

If one chooses the version of Lorentz and Poincaré, all these difficulties disappear.

In The Ghost in the Atom, ${ }^{9}$ Bell says about the EPR paradox (p. 48): "I would say, that the cheapest resolution is something like going back to relativity as it was before Einstein, when people like Lorentz and Poincaré thought that there was an aether - a preferred frame of reference - but that our measuring instruments were distorted by motion in such a way that we could not detect motion through the aether. Now, in that way you can imagine that there is a preferred frame of reference, and in this preferred frame of reference things do go faster than light."

In the same book, ${ }^{9}$ Bohm says about locality: "I would be quite ready to relinquish locality; I think it is an arbitrary assumption." and Basil Hiley says about nonlocality: "If you have an absolute spacetime, or an absolute time, in the background, then you do not get into causal loops. So, the causal paradoxes will not arise."

The entanglement of two particles (let us say two photons) could be realized by the proposed gravitons which move much faster than light.

To determine the speed of gravitons, one reasoning is to consider that the connection between two particles remains even if the two particles are situated at two opposite extremities of our Universe and that our more accurate instruments cannot detect a duration between the measurement of the first particle and the measurement of the second particle, i.e., the duration is smaller than the Planck time $t_{\text {Planck }}=5.4 \times 10^{-44} \mathrm{~s}$.

So we obtain the following speed for the gravitons and the speed of gravitation:

$$
V_{G} \approx \frac{R_{\text {universe }}}{t_{\text {Planck }}}
$$

which gives

$$
V_{G} \approx 13.7 \times 10^{9} \mathrm{AL} / 5.4 \times 10^{-44}=2.4 \times 10^{69} \mathrm{~m} / \mathrm{s}
$$

It is only in Einstein's version (the theory of relativity) that nothing can move faster than light.

\section{VACUUM ENERGY ACCORDING TO THE THEORY OF THE DYNAMIC MEDIUM OF REFERENCE}

According to the theory of the dynamic medium of reference, the "true vacuum" or "perfect vacuum" does not exist.

The gravitons being everywhere in the Universe (and even beyond the visible Universe), a given volume, even intersidereal vacuum, still contains gravitons.

The gravitons constitute a field and form a medium.
The main characteristics of a graviton are its translation speed $\overrightarrow{V_{G}}$ and its rotation speed $\overrightarrow{\omega_{G}}$.

The energy of a unique graviton would be

$$
E_{G}=\alpha \cdot\left\|\overrightarrow{V_{G}}\right\|^{2}+\beta \cdot\left\|\overrightarrow{\omega_{G}}\right\|^{2}
$$

where $\alpha$ and $\beta$ are two constants of proportionality.

Let us suppose that a cube meter of vacuum contains in average $\mathrm{N}_{\mathrm{G}}$ gravitons.

The vacuum energy contained in one cube meter is simply given by the following expression:

$$
\begin{aligned}
E_{\text {vacuum }} & =E_{\text {medium }} \\
& =\sum_{i=1}^{N_{G}} E_{G}(i) \\
& =\sum_{i=1}^{N_{G}}\left(\alpha \cdot\left\|\overrightarrow{V_{G}}\right\|^{2}+\beta \cdot\left\|\overrightarrow{\omega_{G}}\right\|^{2}\right) \\
& =N_{G} \cdot E_{G}=N_{G}\left(\alpha V_{G}^{2}+\beta \omega_{G}^{2}\right),
\end{aligned}
$$

where $E_{G}$ is the average energy of a graviton, $V_{G}$ is the average translation speed of a graviton, and $\omega_{G}$ is the average rotation speed of a graviton.

Remark: the chosen referential to measure the translation speed of the gravitons has a negligible effect because it is moving slower than the speed of light with regard to the Preferred Frame of Reference, and the speed of the gravitons is at least $10^{60}$ times the speed of light.

\section{A. Approximation of the vacuum energy}

We assume that the standard gravitons of the gravitons field of speed $V_{G}$ have a negligible energy of rotation compared to their energy of translation.

We will show that it is the same for the gravitons-spin of speed $V_{G \text { spin }}$ having interacted with the atoms of a massive body.

The energy of a standard graviton is

$E_{1} \approx \alpha V_{G}^{2}=E_{1 T}$

The energy of a graviton-spin is

$$
E_{2}=\alpha V_{G \text { spin }}^{2}+\beta \omega_{G \text { spin }}^{2}=E_{2 T}+E_{2 R} .
$$

The gravitons always keep the same energy, and so we have $E_{1}=E_{2}$, which gives

$$
\begin{aligned}
E_{2 R} & =E_{1 T}-E_{2 T} \\
& =\alpha\left(V_{G}^{2}-V_{G \text { spin }}^{2}\right) \\
& =\alpha V_{G}^{2}\left[1-\left(\frac{V_{G \text { spin }}}{V_{G}}\right)^{2}\right] \\
& =\alpha V_{G}^{2}\left[1-\left(1-\frac{\Delta V_{G}}{V_{G}}\right)^{2}\right] .
\end{aligned}
$$

In a future article, it will be shown that $\Delta V_{G}=$ $V_{G}-V_{G \text { spin }}$ is negligible with regard to $\mathrm{V}_{\mathrm{G}}$. 
So we have $E_{2 R}=E_{1 T}-E_{2 T} \approx 2 \alpha V_{G} \cdot \Delta V_{G}$, whence

$\frac{E_{2 R}}{E_{1 T}} \approx 2 \frac{\Delta V_{G}}{V_{G}}$.

So we have $\mathbf{E}_{\mathbf{2}} \ll \mathbf{E}_{\mathbf{1 T}}$ and

$E_{2} \approx E_{1}=\alpha V_{G}^{2}$

So the energy of rotation of a graviton-spin remains negligible compared to its energy of translation.

So a good approximation of the vacuum energy is

$$
E_{\text {vacuum }} \approx N_{G} \alpha V_{G}^{2} \text {. }
$$

\section{DARK ENERGY ACCORDING TO THE THEORY OF THE DYNAMIC MEDIUM OF REFERENCE}

Within the theory of the dynamic medium of reference, the far galaxies are carried along by the medium (the gravitons field) and the speed of the galaxies corresponds to the average speed of the moving medium seen by an observer on the Earth which is given by the following formula:

$$
\underset{C_{G / \text { Earth }}}{\longrightarrow}=\frac{\sum_{i=1}^{N_{G}} \overrightarrow{V_{G / \text { Earth }}}}{N_{G}} .
$$

The dark energy would correspond to the dragging energy of the gravitons contained in the medium.

Thus, the dark energy corresponding to one cube meter of medium, containing $\mathrm{N}_{\mathrm{G}}$ gravitons, would be given by the following formula:

$$
E_{\text {dark }}=E_{C C}=N_{G} \cdot \alpha \cdot\left\|\overrightarrow{C_{G / \text { Earth }}}\right\|^{2} .
$$

This energy is also called $E_{C C}$ because it corresponds to the energy due to the cosmological constant of the general relativity properly interpreted and assessed.

To determine the value of $\left\|\overrightarrow{C_{G / \text { Earth }}}\right\|$, we have to use Hubble's law ${ }^{1,2} V=H . D$, which brings in the famous Hubble's constant $\mathrm{H}$.

The current observations approximately match toward a value around $70(\mathrm{~km} / \mathrm{s}) / \mathrm{Mpc}$. This means that a galaxy located at $1 \mathrm{Mpc}$ (about $3.26 \times 10^{6}$ light years) from the observer is going away by the fact of the expansion of the Universe (and thus without taking into account the proper movement of the object, negligible at very high distances) with a speed of $70 \mathrm{~km} / \mathrm{s}$.

The Planck mission ${ }^{10}$ allowed to calculate Hubble's constant, which is a recession speed of $67.8 \mathrm{~km} / \mathrm{s}$ and per mega parsec.

A galaxy located at $D_{\text {galaxy }}=13.7 \times 10^{9}$ light years $(4200 \mathrm{Mpc})$ is going away at a speed of

$$
V_{\text {galaxy }}=H . D=67.8 \times 4200=284760 \mathrm{~km} / \mathrm{s} .
$$

This speed is very close to the speed of light, so it is possible to make the following approximation:

$$
\left\|\overrightarrow{C_{G / \text { Earth }}}\right\| \approx V_{\text {galaxy }} \approx c .
$$

\section{COMPARISON BETWEEN VACUUM ENERGY AND DARK ENERGY}

Taking the ratio between the vacuum energy and the dark energy, we find the following important relation:

$$
\frac{E_{\mathrm{vacuum}}}{E_{\mathrm{dark}}}=\frac{\alpha V_{G}^{2}+\beta \omega_{G}^{2}}{\alpha\left\|\overrightarrow{C_{G / \text { Earth }}}\right\|^{2}}=\left(\frac{V_{G}}{C_{G}}\right)^{2}+\frac{\beta}{\alpha}\left(\frac{\omega_{G}}{C_{G}}\right)^{2} .
$$

Taking into account the approximation of the vacuum energy in Sec. V, we obtain

$$
\frac{E_{\mathrm{vacuum}}}{E_{\mathrm{dark}}} \approx\left(\frac{V_{G}}{C_{G}}\right)^{2}
$$

This can provide us an estimation of the average speed of the gravitons using the approximation $C_{G} \approx V_{\text {galaxy }}$,

$$
V_{G}=C_{G} \sqrt{\frac{E_{\mathrm{vacuum}}}{E_{\text {dark }}}} \approx V_{\text {galaxy }} \sqrt{\frac{E_{\mathrm{vacuum}}}{E_{\text {dark }}}} .
$$

The calculation gives

$$
V_{G} \approx 2.8 \times 10^{8} \sqrt{\frac{10^{113}}{10^{-9}}} \approx 2.8 \times 10^{69} \mathrm{~m} / \mathrm{s} .
$$

In Sec. IV B, we have showed that

$$
V_{G} \approx \frac{R_{\text {universe }}}{t_{\text {Planck }}} \approx 2.4 \times 10^{69} \mathrm{~m} / \mathrm{s} \text {. }
$$

The two values of the speed of the gravitons obtained with two totally different formulae are in good agreement.

Remark: it is possible to determine the product $\mathrm{N}_{\mathrm{G}} \cdot \alpha$ between the number of gravitons contained in one cube meter "of vacuum" and the constant of proportionality $\alpha$,

$$
N_{G} . \alpha \approx \frac{E_{\mathrm{vacuum}}}{V_{G}^{2}} \approx \frac{E_{\mathrm{dark}}}{C_{G}^{2}} \approx \frac{10^{-9}}{\left(2.8 \times 10^{8}\right)^{2}} \approx 10^{-26} \mathrm{~J} \mathrm{~m}^{-2} \mathrm{~s}^{2} .
$$

\section{ANALOGY WITH A VOLUME OF AIR}

We consider a volume of air and we suppose that this volume is pushed by a wind of speed $C_{W}$.

It is possible to consider two types of energy associated with this volume of air:

- the "group energy" of the volume of air,

- the energy of all the molecules composing the volume of air.

\section{A. "Group energy" of the volume of air (analogy with dark energy)}

The "group energy" of the volume of air is given by the following formula: 


$$
E_{G}=\frac{1}{2} M \cdot C_{W}^{2}=\frac{1}{2} N \cdot m \cdot C_{W}^{2} .
$$

$C_{W}$ represents the speed of the overall movement of the volume of air which is equal to the speed of the wind.

$M$ represents the mass of the volume of air which is equal to $N$ times the mass $m$ of a molecule of air where $N$ represents the number of molecules of air contained in the volume.

\section{B. Energy of all the molecules contained in the volume of air (analogy with the vacuum energy)}

The energy of the $\mathrm{N}$ molecules of mass $m$ contained in the volume of air is given by the following formula:

$$
E_{\text {total }}=\frac{1}{2} N \cdot m \cdot u^{2}
$$

$u$ is the root-mean-square speed for which the kinetic theory of gases gives the following expression:

$$
u=\sqrt{\left\langle v^{2}\right\rangle}=\sqrt{3 \frac{k_{B} \cdot T}{m}} .
$$

At ambient temperature (around $300 \mathrm{~K}$ ), this speed can reach several thousands of meters per second and is much greater than the "group speed" of the volume of air.

Here again, the ratio between the two energies is equal to the square of the ratio of the two speeds,

$$
\frac{E_{\text {total }}}{E_{G}}=\left(\frac{u}{C_{W}}\right)^{2}
$$

\section{CONCLUSION}

The theory of the dynamic medium of reference allows raising the mystery about the huge divergence between the vacuum energy and the dark energy called the "vacuum catastrophe.'

From a physical point of view, the difference between vacuum energy and dark energy is as follows:

- The vacuum energy is the energy of the gravitons of the gravitons field.

- The dark energy is the energy of the flux of the medium on a very large scale, that of the Universe.

From a mathematical point of view the enormous difference between vacuum energy and dark energy can be explained by the following two formulas:

$$
\text { - } E_{\mathrm{vacuum}} \approx N_{G} \alpha V_{G}^{2} \approx \alpha \cdot N_{G} V_{G}^{2} \approx \alpha \sum_{1}^{N_{G}}\left\|\overrightarrow{V_{G}}\right\|^{2},
$$

$$
\begin{aligned}
\text { - } E_{\text {dark }} & =N_{G} \alpha\left\|\overrightarrow{C_{G}}\right\|^{2}=\alpha N_{G}\left\|\frac{\sum_{1}^{N_{G}} \overrightarrow{V_{G}}}{N_{G}}\right\|^{2} \\
& =\alpha \frac{\left\|\sum_{1}^{N_{G}} \overrightarrow{V_{G}}\right\|^{2}}{N_{G}} .
\end{aligned}
$$

If all the gravitons were going in the same direction, it is easy to verify that the two energies would be equal. As gravitons move in all possible directions and $N_{G}$ and $V_{G}$ are very large, the term $\sum_{1}^{N_{G}}\left\|\overrightarrow{V_{G}}\right\|^{2}$ is much greater than the term $\left\|\sum_{1}^{N_{G}} \overrightarrow{V_{G}}\right\|^{2}$ and, therefore, the vacuum energy is much greater than the dark energy.

Knowing the values of the vacuum energy and of the dark energy makes it possible to deduce the speed of the gravitons by the formula $V_{G}=C_{G} \sqrt{\left(E_{\text {vacuum }} / E_{\text {dark }}\right)} \approx$ $V_{\text {galaxy }} \sqrt{\left(E_{\text {vacuum }} / E_{\text {dark }}\right)}$ which is in good agreement with the value obtained by the formula $V_{G} \approx R_{\text {universe }} / t_{\text {Planck }}$.

Finally, about the acceleration of the galaxies moving away which corresponds to the accelerating expansion of the universe, this one is well understood within the frame of the theory of the dynamic medium of reference because it is not due to a new force or repulsive energy but it is due to the same physical phenomenon which implies that matter is dragged by the gravitons field, the "dragging speed" being the vectorial average of the speed vectors of the gravitons coming from all the directions comprising from the outside of the visible Universe.

It seems quite natural that the fundamental average vector $\overrightarrow{C_{G}}=\sum_{i=1}^{N_{G}}\left(\overrightarrow{V_{G}}\right) / N_{G}$, where the contribution of all the gravitons counterbalance may vary along the time and the expansion of the Universe.

${ }^{1}$ M. Holson, G. Efstathiou, and A. Lasenby, Relativité Générale (General Relativity) (De Boeck, Paris, France, 2010).

${ }^{2}$ P. J. E. Peebles and B. Ratra, Rev. Mod. Phys. 75, 559 (2003).

${ }^{3}$ P. J. Steinhardt and N. Turok, Science 312, 1180 (2006).

${ }^{4}$ P. W. Milonni, The Quantum Vacuum (Academic Press, Inc., Cambridge, MA, 1994).

${ }^{5}$ L. de la Peña and A. M. Cetto, The Quantum Dice: An Introduction to Stochastic Electrodynamics (Springer Science and Business Media, Berlin, 2013).

${ }^{6}$ O. Pignard, Phys. Essays. 32, 422 (2019).

${ }^{7}$ H. Poincaré, Science et Méthode (Science and Method) (Flammarion, Paris, France, 1908).

${ }^{8}$ O. Pignard, Phys. Essays. 33, 395 (2020).

${ }^{9} \mathrm{P}$. Davies and J. Brown, The Ghost in the Atom (Cambridge University Press, Cambridge, UK, 2000).

${ }^{10}$ Planck Collaboration, Astron. Astrophys. 641, A6 (2020). 